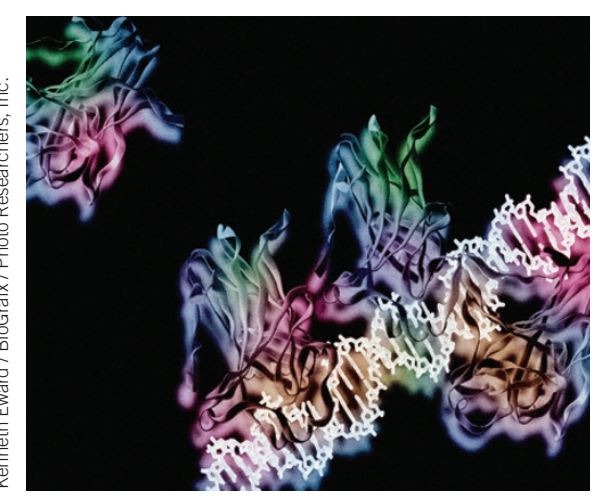

that are involved in controlling the apoptotic, cell cycle arrest and senescence functions of p53. Cells and tissues from mice expressing this mutated p53 showed that the mutations abrogated these cellular functions in response to DNA damage, but, surprisingly, the animals were protected from lymphoma development. The expression of genes involved in modulating mitochondrial respiration and limiting glycolysis and oxidative damage were still induced by p53 in these mice, suggesting that p53 may prevent tumorigenesis by regulating metabolism.

Other, as yet unknown, p53 functions may be also involved in cancer suppression, and future research should aim to elucidate the exact antitumor effect of p53-mediated metabolic and antioxidant functions before new therapeutic approaches can be designed to modulate these tumor suppressor pathways. $-C P$

\section{METABOLIC DISORDERS}

\section{A route to leanness}

A population of hypothalamic neurons expressing the Agouti-related peptide (AgRP) has been shown to be important in the induction of feeding. Although the hormones leptin and insulin act on these neurons, knocking out either leptin or insulin signaling in AgRP neurons does not seem to affect energy homeostasis. Now, Hongxia Ren et al. show that disabling both these pathways in mice by knocking out a central transcriptional mediator, FoxO1, in AgRP neurons can improve metabolism, resulting in lean mice that eat less (Cell 149, 1314-1326).

The researchers then performed microarray analyses of the AgRP hypothalamic neurons with or without FoxO1 and found that the G-coupled protein receptor Gpr17 was downregulated in FoxO1-deficient neurons. In cell culture studies, they found that FoxO1 directly bound the Gpr17 gene and increased its expression. When they administered Gpr17 agonists into the brain ventricles of mice, this increased feeding. A Gpr17 antagonist decreased feeding, suggesting that targeting this pathway could be used to treat obesity in humans. $-E C$

\section{- INFECTIOUS DISEASES \\ Turning the tide against TB}

Multidrug-resistant tuberculosis, accounting for nearly $5 \%$ of cases, is difficult and expensive to treat. A recent clinical trial reports positive results for a new drug, delamanid, in people with this form of tuberculosis (N. Engl. J. Med. 366, 2151-2160).

Delamanid, an inhibitor of mycolic acid synthesis, has previously been shown to have antituberculosis activity in both in vitro and in vivo preclinical studies. Gler et al. tested delamanid at two doses in a randomized, placebo-controlled trial. A total of 321 patients with multidrug-resistant tuberculosis were treated with delamanid, as compared to 160 patients treated with placebo, with all patients receiving a background drug regimen. After 2 months of treatment, the two delamanid-treated groups had a higher proportion of sputum cultures that were negative for mycobacterial growth. However, a dose response was not observed. The drug seemed to be well tolerated, although it was associated with an effect on cardiac electrical activityQT interval prolongation.

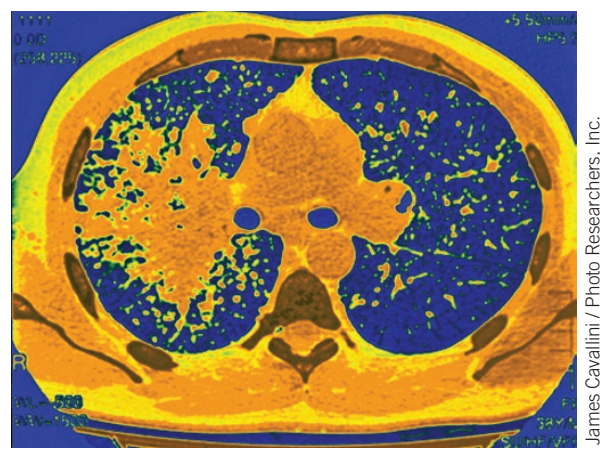

Effects on sputum culture growth have previously been associated with long-term outcomes of tuberculosis treatments, but longer clinical trials of delamanid will be needed. In addition, it will be important to determine how delamanid can be used most effectively in combination with other antituberculosis agents. $-M B$

Written by Victoria Aranda, Michael Basson,

Eva Chmielnicki, Juan Carlos López, Carolina Pola and Meera Swami.

\section{New from NPG}

The mutational landscape of lethal castration-resistant prostate cancer Grasso, C.S. et al. Nature doi:10.1038/ nature11125 (20 May).

Exome sequencing identifies recurrent SPOP, FOXA1 and MED12 mutations in prostate cancer

Barbieri, C.E. et al. Nat. Genet. 44, 685-689 (2012).

Two studies use exome sequencing to investigate the genetic basis of prostate cancer. Barbieri et al. identify recurrent mutations in multiple genes in prostate cancer, showing that SPOP contains the most frequent recurrent mutations. Grasso et al. define mutations in castrationresistant prostate cancer, suggesting a mechanism through which these cancers become resistant to antiandrogen therapy.

F-box protein FBXL19-mediated ubiquitination and degradation of the receptor for IL-33 limits pulmonary inflammation

Zhao, J. et al. Nat. Immunol. doi:10.1038/ ni.2341 (3 June)

The cytokine IL-33 is important in lung inflammatory conditions such as asthma. The interaction between IL-33 and its receptor $\mathrm{ST} 2 \mathrm{~L}$ is shown to be regulated by an intracellular protein FBXL19, which binds and degrades ST2L. Therapeutic modulation of this axis might be one way to reduce lung inflammation.

Autism spectrum disorder susceptibility gene TAOK2 affects basal dendrite formation in the neocortex Calderon de Anda, F. et al. Nat. Neurosci. doi:10.1038/nn.3141 (10 June).

The authors find that the gene encoding thousand-and-one amino acid 3 kinase (TAOK2), which has been associated with susceptibility to autism spectrum disorders, is essential for dendrite morphogenesis. They also define a pathway involving TAOK2, neuropilin 1 , semaphorin $3 A$ and JNK that regulates the development of basal dendrites in the cortex.

Structural basis of evasion of cellular adaptive immunity by HIV-1 Nef Jia, X. et al. Nat. Struct. Mol. Biol. doi:10.1038/nsmb.2328 (17 June).

The authors characterize the structure of a complex of the HIV-1 restriction factor Nef, the class I major histocompatibility complex and a subunit of the clathrin adaptor protein complex 1 . This allowed them to show how Nef can modulate host membrane trafficking, enabling HIV-1 to evade the adaptive immune system. 